\title{
Características y prevalencia de los eventos adversos presentados en la Clínica del Posgrado de Periodoncia de la Facultad de Odontología de la Pontificia Universidad Javeriana durante el período 2011 - $2012^{*}$
}

Characteristics and Prevalence of Adverse Events Reported at the Postdoctoral Periodontal Clinic of the Pontifical Javeriana University Dental School between 2011 and 2012

Daissy Julieth Tafur Gallego ${ }^{1}$

Gina Paola Ramírez Vélez ${ }^{2}$

DOI: https://doi.org/10.11144/Javeriana.uo35-75.cpea Redalyc: http://www.redalyc.org/articulo.oa? id $=231249121010$

César Andrés Cárdenas Penagos ${ }^{3}$

Juan Jaime Serrano Álvarez a

Pontificia Universidad Javeriana, Colombia

j-serrano@javeriana.edu.co

Ana Lucia Sarralde Delgado

Pontificia Universidad Javeriana, Colombia

Fecha de recepción: 27 Marzo 2016

Fecha de aprobación: 23 Diciembre 2016

Sandra Patricia Camacho Peña Pontificia

Universidad Javeriana, Colombia

Adriana Rodríguez Ciódar

Pontificia Universidad Javeriana, Colombia

Juliana González Moncada

Pontificia Universidad Javeriana, Colombia

\section{Resumen:}

Antecedentes: El aumento en la incidencia de los eventos adversos derivados de la práctica clínica a causa de errores potencialmente prevenibles ha generado preocupación en la Organización Mundial de la Salud, debido a su gran impacto en la salud de los pacientes. Por lo tanto, es necesario establecer medidas que garanticen la atención segura del paciente por parte de las instituciones educativas que proveen servicios de salud. Propósitos: Identificar, caracterizar y documentar la frecuencia de los eventos adversos en el área de periodoncia, detectados en las historias clínicas de los pacientes que asistieron a la Clínica de Posgrado de Periodoncia de la Facultad de Odontología de la Pontificia Universidad Javeriana en el periodo 2011-2012. Métodos: Se realizó un estudio descriptivo en el que se analizaron 929 historias clínicas para identificar y caracterizar los eventos adversos en periodoncia. Se evaluaron 3886 procedimientos durante 2011 y 2012. Resultados: Los eventos adversos en periodoncia encontrados fueron 15 de 929 historias. Los eventos adversos no clínicos prevenibles se identificaron en un 9,43\% y los eventos adversos clínicos prevenibles lo fueron en un 16,9\%. Sin embargo, se detectó que, de los 1738 procedimientos quirúrgicos reportados, solo 406 casos recibieron control posquirúrgico. Conclusión: La frecuencia observada de eventos adversos prevenibles en periodoncia indica una baja tasa de reporte y un subregistro de estos a partir de la falencia en el registro de controles posquirúrgicos. Resulta por ello importante establecer mecanismos de reporte y seguimiento.

Palabras clave: evento adverso, historia clínica, manejo del riesgo, periodoncia, seguimiento del paciente, seguridad del paciente. Áreas Temáticas: administración en salud; periodoncia.

Notas de autor:

\footnotetext{
1,2,3 Práctica privada.

a Autor de correspondencia. Correo electrónico: j-serrano@javeriana.edu.co
} 


\begin{abstract}
:
Background: The increase of incidence of adverse events because of preventable clinical errors has alarmed the World Health Organization due to their impact on patients' health. Therefore, it is mandatory to establish procedures to guarantee patient safety among higher education institutions that provide health care services. Purposes: To identify, characterize, and determine the frequency of adverse events detected in the dental records of patients that attended the Postgraduate Clinic of Periodontics of the Pontifical Javeriana University between 2011 and 2012. Methods: In this descriptive study, 929 dental records were analyzed to identify adverse events in Periodontics. 3.886 periodontal procedures were evaluated in 2011 and 2012 . Results: 15 adverse events in periodontics were analyzed. $9.43 \%$ preventable non-clinical adverse events and $16.9 \%$ preventable clinical adverse events were identified. However, of the 1738 surgical procedures reported, only 406 cases had a follow-up. Conclusion: The frequency of observed preventable periodontal adverse events indicates a low report rate. The underreport of the follow-up information during postoperative controls, makes it mandatory to create reporting and follow-up procedures. Escuchar Leer fonéticamente.
\end{abstract}

Keywords: adverse effects, patient maintenance, medical record, patient safety, periodontics, risk management.

Thematic Fields: health care management; periodontics.

\title{
INTRODUCCIÓN
}

La prestación y calidad de los servicios odontológicos debe promover y garantizar la atención segura del paciente, a través del cumplimiento de los estándares de bioseguridad, los protocolos basados en la evidencia científica y las reglamentaciones estipuladas por las instituciones gubernamentales nacionales y mundiales. Sin embargo, el desenlace negativo o positivo de un procedimiento clínico puede derivarse de condiciones administrativas o prácticas anteriores, durante el tratamiento o posteriores a este [1].

En consecuencia, el profesional de la salud debe tener claridad en la terminología de la atención segura del paciente, de acuerdo con la situación clínica que se presente, de forma que una vez sucedan complicaciones, esté en capacidad de clasificarlas y brindar el seguimiento apropiado. Un ejemplo de lo anterior son complicaciones correspondientes a un daño o resultado clínico no esperados, no atribuibles a la atención en salud, sino a la enfermedad o a las condiciones propias del paciente. En contraste, el evento adverso (EA) ocurre como resultado de una atención insegura en salud que de manera no intencional produjo daños en el paciente, siendo más atribuible a condiciones de la atención y no a la enfermedad subyacente $[1,2,3]$.

No obstante, el aumento en la incidencia de los EA derivados de la práctica clínica, a causa de errores potencialmente prevenibles ha generado preocupación en la Organización Mundial de la Salud, por el impacto de estos en la salud de los pacientes. Es necesario establecer medidas que garanticen la atención segura $[1,4]$. En este contexto, en Colombia se estableció la obligatoriedad de la vigilancia de los EA en toda institución prestadora de servicios de salud, a través del Sistema Obligatorio de Garantía de Calidad en Salud y la Resolución 1446 del 2006. Es una estrategia para disminuir y prevenir la ocurrencia de tales eventos y garantizar una atención con calidad a través de la identificación, reporte y seguimiento de EA [5,6]. En respuesta, el Posgrado de Endodoncia de la Facultad de Odontología de la Pontificia Universidad Javeriana (FOPUJ) de Bogotá, acatando las políticas de calidad en atención de salud, inició el proceso de análisis de EA en 2007. En ese entonces, se encontraron 306 EA en 464 historias clínicas, con una alta frecuencia de EA prevenibles en un 81,3\% de los casos [7,8]. Este proceso continuó con el Posgrado de Periodoncia de la misma universidad y constituye así una línea de investigación.

A pesar de los grandes esfuerzos legislativos tanto nacionales como mundiales para monitorear y controlar los EA, los clínicos los manejan poco, debido a que los conceptos no se interpretan y desarrollan claramente. Esto se evidencia en la literatura científica, más específicamente en la especialidad de la periodoncia, en la que los estudios de identificación y seguimiento de los EA no se han documentado ampliamente y muchas imprecisiones se observan en la distinta terminología relacionada. Faggion y 
Giannakopoulos [9] llevaron a cabo una revisión sistemática de la literatura de ensayos clínicos aleatorizados, de 2012 a 2013, sobre los efectos terapéuticos en gingivitis y periodontitis en humanos, para evaluar la calidad de los reportes de EA bajo los Estándares Consolidados de Reporte de Estudios (CONSORT, por su sigla en inglés: Consolidated Standards of Reporting Trials). Hallaron que de 392 artículos evaluados, solo 4 cumplieron con este criterio de inclusión. Asimismo, la estandarización de reportes de EA en ensayos clínicos aleatorizados para periodoncia e implantología dental aún no ha sido evaluada, lo cual indica una deficiencia en su identificación y seguimiento. Los autores concluyeron que se requiere una mejora en las pautas de informe de los EA.

En la literatura también se evidencia una comprensión equivocada entre iatrogenia y EA. Sgolastra y colaboradores [10] efectuaron un metanálisis de la efectividad del metronidazol como terapia coadyuvante al raspaje y alisado radicular. Identificaron 3 artículos de 517 que mencionaron EA asociados a medicamentos, como gastritis y náuseas, lo cual constituye una iatrogenia: daño producido por una droga o procedimiento bajo una condición correcta y criterio justo y que, pese a ello, se producen molestias o enfermedad, según los términos de atención segura del paciente [2]. Adicionalmente, es importante comprender que la causa del EA no corresponde exclusivamente a fallas activas por una toma de decisión inadecuada del profesional. También puede ocurrir como consecuencia de una cadena de deficiencias en las herramientas administrativas de respaldo clínico. De esta forma, el error humano constituye una fracción de la falla en la atención segura del paciente $[11,12,13,14]$.

Con lo anterior, dada la literatura escasa y específica acerca de la asociación de prácticas inseguras en el área de periodoncia que lleven al desarrollo de EA y a su inadecuada definición, categorización y tipifi cación, surge la necesidad de identificar los EA presentados en la Clínica del Posgrado de Periodoncia a través de la revisión de historias clínicas, en busca de contribuir al mejoramiento de las políticas de seguridad del paciente en el Departamento de Periodoncia de la Pontificia Universidad Javeriana.

Adicionalmente, la legislación colombiana cada día es más exigente con respecto al cumplimiento de las políticas de seguridad del paciente, a través del Ministerio de Salud y Protección Social, que expidió los decretos 2309 de 2002 y 1011 de 2006, cuyo fin último es controlar los EA por medio del Sistema Obligatorio de Garantía de Calidad en Salud, a efectos de realizar auditorías para el mejoramiento en la atención en salud; por lo tanto, siendo la FOPUJ una institución prestadora de servicios de salud, no es ajena al contexto legal nacional, lo que evidencia la necesidad del desarrollo de modelos efectivos de seguimiento de EA [15].

Para ello, esta investigación pretendió identificar, caracterizar y documentar la frecuencia de los EA en el área de periodoncia, detectados en las historias clínicas de los pacientes que asistieron a la Clínica del Posgrado de Periodoncia de la FOPUJ en el periodo 2011-2012.

\section{MATERIALES Y MÉTODOS}

En este estudio observacional descriptivo se analizaron 929 historias clínicas de pacientes que asistieron al Posgrado de Periodoncia en la FOPUJ entre 2011 y 2012, con una totalidad de procedimientos clínicos quirúrgicos y no quirúrgicos de 3886, el cual fue aprobado por el Comité de Ética de la FOPUJ.

Los criterios de inclusión fueron todas las historias clínicas de pacientes atendidos en las clínicas del Posgrado de Periodoncia de la Pontificia Universidad Javeriana, en el periodo 2011-2012, con autorización del docente y sin este, con reporte de tratamientos no finalizados, letra ilegible, borrosa o con la tinta corrida que aunque dificulte la lectura de los datos, sí se detecta un desenlace no deseado en una historia con las características mencionadas, que se incluyeron como defecto administrativo. No se excluyó ninguna historia.

Para el desarrollo de la prueba, inicialmente, se recolectó información en un formato construido basándose en hallazgos de la literatura y el apoyo de odontólogos clínicos y administrativos, todos docentes de la FOPUJ con conocimiento en los modelos de gestión en seguridad del paciente. En consecuencia, debido a que en el Posgrado de Periodoncia no se habían realizado investigaciones sobre atención insegura, se determinó que en este estudio se iban a registrar como situaciones anómalas aquellos desenlaces no deseados clínicos en tratamientos no quirúrgicos y quirúrgicos y fallas en el proceso administrativo, con el fin de clasificarlas posteriormente bajo la terminología de atención segura del paciente (figura 1). 


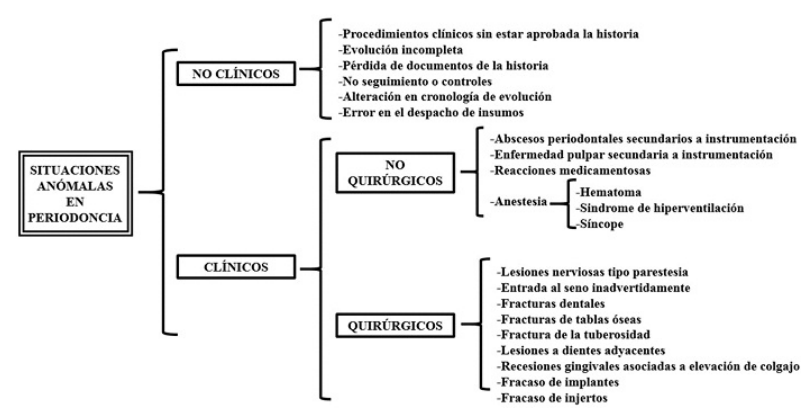

FIGURA 1

Clasificación de situaciones anómalas en periodoncia basadas en la evidencia científica y experiencia de docentes

Una vez se cumplió la revisión de 441 historias clínicas ( $48 \%$ ) por parte de cada uno de 3 investigadores, se convocó a una reunión de expertos, compuesta por 7 especialistas: 4 periodoncistas y 3 administrativos, parte del grupo de apoyo de docentes para conformar la sábana de situaciones anómalas, con mínimo 15 años de experiencia clínica y docencia, a quienes se les presentaron las situaciones anómalas identificadas, con el fin de que las evaluaran para su tipificación dentro de la taxonomía de política del paciente en atención en salud.

Los datos obtenidos se utilizaron como parámetro para precategorizar las siguientes situaciones anómalas, detectadas durante la finalización de recolección de datos de las historias clínicas faltantes. Posteriormente, se presentaron los nuevos casos ( $52 \%$ de historias clínicas restantes) a los mismos expertos del análisis inicial, cuyos resultados se presentaron con estadística descriptiva y se ofrece una clasificación de los EA con base en la práctica no clínica y clínica en quirúrgicos y no quirúrgicos.

Adicionalmente de las situaciones identificadas como EA, se registró el número de historia clínica, la edad, el género, la presencia de componentes sistémicos, el diligenciamiento de historia clínica y anamnesis, el procedimiento realizado, la autorización del docente, el EA presentado, el manejo y seguimiento y las observaciones en el sistema para posterior evaluación, si llegara a ser requerido.

\section{RESULTADOS}

Inicialmente, se aclaró la distribución de los 3886 procedimientos realizados entre 2011 y 2012, divididos en 1) tratamientos clínicos no quirúrgicos, que incluyeron desde la valoración hasta la ambientación periodontal a campo cerrado, correspondiendo a 2148, y 2) en clínicos quirúrgicos, de los cuales se registraron 1738 (44,8 \%) y que solo reportan 406 controles posquirúrgicos, correspondiendo al 23,3\%. Se encontró un alto porcentaje de deficiencia en el seguimiento del paciente sometido a una cirugía y, por lo tanto, pérdida de información sobre las secuelas positivas o negativas de cada uno de los casos (figura 2).

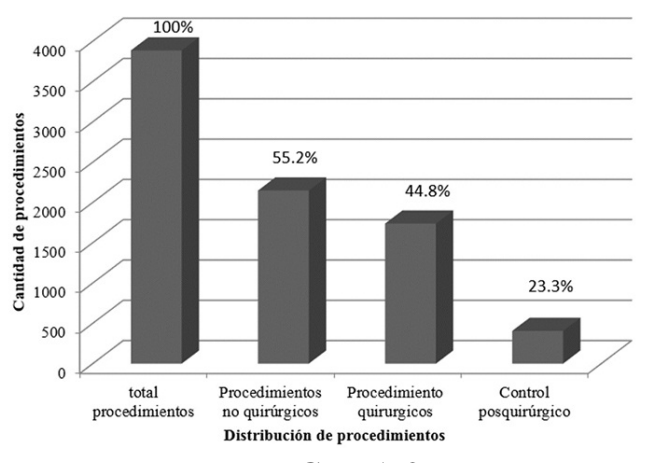

FIGURA 2

Distribución total de procedimientos con el comparativo entre no quirúrgicos y quirúrgicos

La identificación de situaciones anómalas tanto clínicas como no clínicas en periodoncia mostró un 5,7 $\%$, aunque ninguna fue descrita en la historia clínica en condición de EA o algún otro término como complicación, iatrogenia, error, entre otros. Ello demuestra un bajo porcentaje de reporte y descripción en 
las evoluciones, dado que de 929 historias clínicas, solo se detectaron 53 casos (5,7\%) en los que se presentó alguna anomalía durante la fase de atención o posquirúrgica. Los expertos evaluaron estas situaciones para tipificarlas, y a partir de las circunstancias identificadas, cada uno de los casos fue representado dentro de un $100 \%$ (figura 3). Se observó una variabilidad en la taxonomía identificada, por lo cual se describirán las condiciones analizadas para cada evento y por qué su consideración en la diferente terminología.

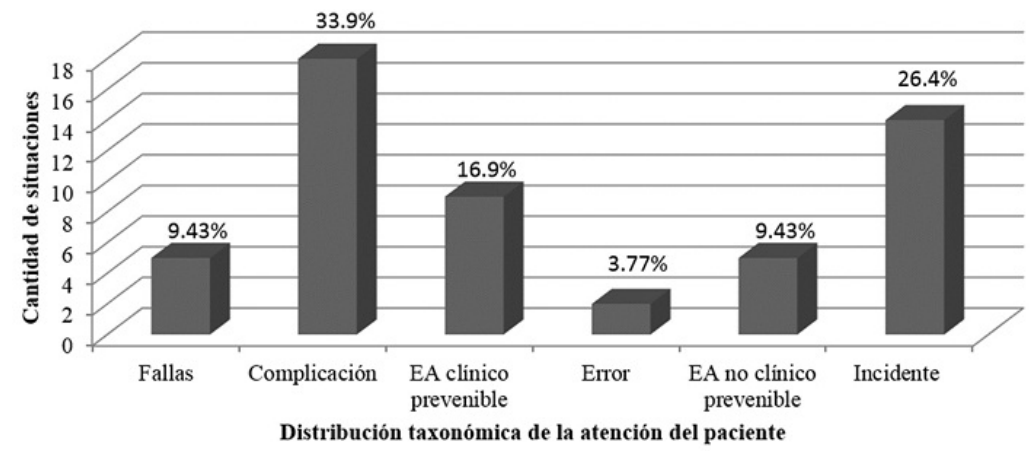

FIGURA 3

Tipificación de situaciones anómalas detectadas

Se encontró una mayor frecuencia de complicaciones observada en 18 casos (33,9\%), que corresponden a daños o resultados clínicos no esperados, no atribuibles a la atención en salud, sino a la enfermedad o las condiciones propias del paciente [2] (figura 4).

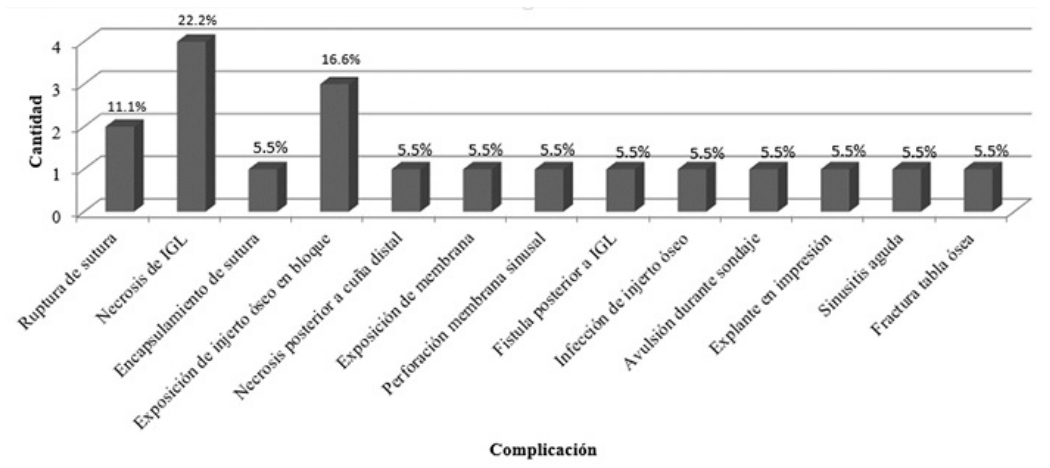

FIGURA 4

Tipificación de complicaciones

En este análisis predominó la necrosis de injerto gingival libre, seguida por la exposición de injerto óseo en bloque y pérdida de puntos de sutura. Las demás condiciones se presentaron una sola vez, debido a que cada suceso se estima que puede acontecer independientemente del operador y se origina a partir de la evolución de la patología o respuesta del paciente al tratamiento; por ende, se establecen como riesgos al paciente previo a la realización del procedimiento y cuando se presentan, pueden llevar a su fracaso nulo, parcial o total. Sin embargo, es pertinente aclarar la justificación de la complicación con respecto al caso de la avulsión dental durante el sondaje, debido a que en los hallazgos del periodontograma, evolución y consentimiento informado se registra una movilidad grado III. Se notifica entonces al paciente y al familiar de esta condición y sus consecuencias, dada la extensión de la afectación.

Con respecto al explante, sucedió durante la toma de impresión en el Posgrado de Rehabilitación Oral, el cual precisó reintervención quirúrgica por parte del Posgrado de Periodoncia. Se determinó por la pérdida de fijación del implante dental, con presencia de las cuatro paredes óseas, sin hallazgo de proceso infeccioso. En el caso de la sinusitis aguda, sucedió posterior a elevación del piso del seno maxilar, sin registro de ruptura de la membrana de Schneider y durante los controles se refirió sintomatología. Los casos continuaron con remisión al servicio de otorrinolaringología, donde se realizó el diagnóstico y siguió el manejo por esta área. 
Con relación a las fallas que ocurren frente a una deficiencia para realizar una acción prevista según lo programado o la utilización de un plan incorrecto, 4 casos se relacionaron con fracaso en el aumento de corona clínica por la falta de temporalización que llevó a un sobrecrecimiento de la encía y ello precisó reintervenir quirúrgicamente al paciente. Por otro lado, se reportó un caso de falla en el seguimiento de injerto gingival libre, el cual se sondeó a las dos semanas de su realización. Esto consiste en un procedimiento inadecuado en un tejido en proceso de cicatrización, dado que se debe esperar 12 semanas $[1,2]$.

En cuanto a los errores, aquellos que surgen de un concepto equivocado o juicio falso y llevan a una acción desacertada [1,2], se identificó el primero durante una segunda fase quirúrgica de implante en la que no adaptó el pilar de cicatrización, por lo que se posicionó el tornillo cobertor, se cerró el colgajo y no se describió por qué no se pudo colocar; sin embargo, en rehabilitación oral aparecen evoluciones que reportan continuidad en la restauración y por lo cual no se produjo daño. El segundo error lo constituye un aumento de corona clínica; luego, el diente presentó un aumento de movilidad, relación corona-raíz invertida y exposición de furca, lo cual indicaba su exodoncia. Adicionalmente, no se detectó descripción o análisis radiográfico previo al procedimiento.

Por otro lado, la caracterización de incidentes se definió como una circunstancia que sucede en una atención clínica insegura a un paciente que no le genera daño, pero que en su ocurrencia se incorporan fallas $[1,2]$, por lo cual se catalogaron 14 , dados por la ausencia de controles posquirúrgicos y falta de aprobación mediante la firma y el sello del docente en anexos o evoluciones. Ello representa fallas potenciales que aun cuando no lesionaron al paciente, su realización sin la aprobación y dirección del profesor en una institución - cuyo servicio se basa en docencia asistencial- pudo ocasionar algún perjuicio en el usuario o a la entidad universitaria (figura 3).

Para la identificación de EA se clasificaron en 5 no clínicos prevenibles y en 9 clínicos prevenibles, detectados en 14 casos (26,33 \%; figura 3) no reportados en la historia clínica bajo la denominación de EA, sino como hallazgos durante las citas de seguimiento. Al evaluar los EA no clínicos prevenibles, que corresponden al 9,43\% (figura 3), se tomaron en cuenta las circunstancias tipificadas como EA clínico prevenible, es decir, se presentó EA clínico y no clínico prevenible simultáneamente, al encontrarse falencias en la falta de descripción de los procedimientos y controles posquirúrgicos realizados, como cambios en la cronología de las evoluciones, lo que dificulta adicionalmente la comprensión del proceso del paciente. Igualmente, de los casos registrados, se encontró que más de una variable sucedió en la misma historia, lo que representa mayores obstáculos en su lectura (figura 5). Las anteriores se constituyen como situaciones prevenibles, dado que conciernen a fallas humanas en el proceso administrativo y pueden ser corregidas y supervisadas.

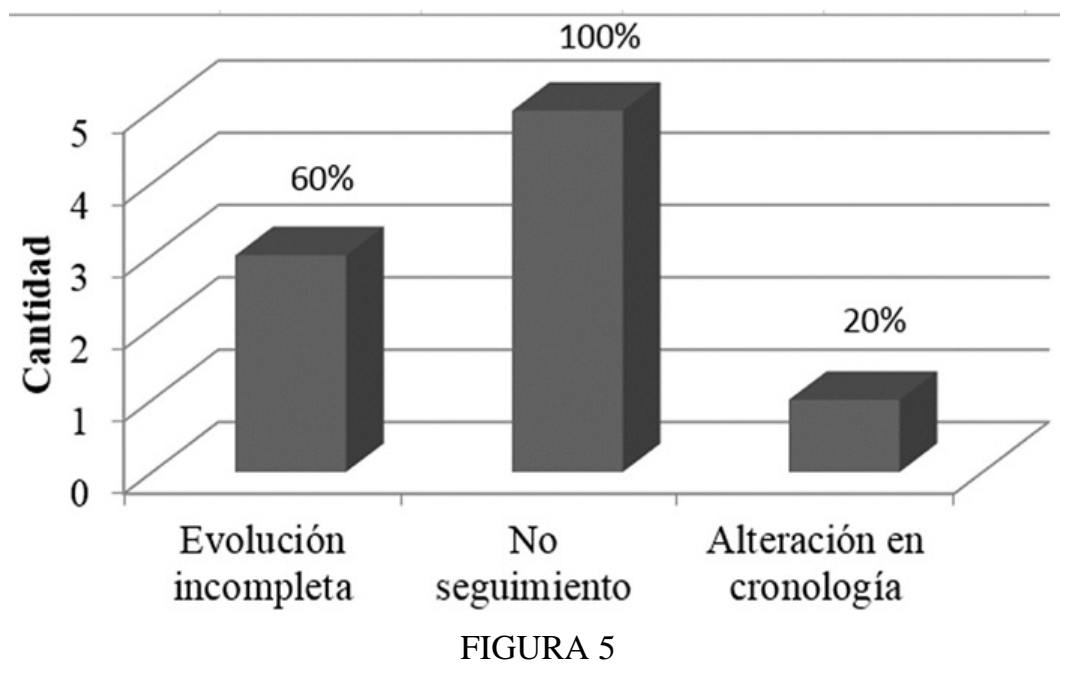

Caracterización de eventos adversos no clínicos prevenibles 
Dentro de la evaluación no se identificaron EA clínicos no quirúrgicos, a pesar de que se realizaron 2148 procedimientos que incluían diligenciamiento de historia clínica de primera vez, reevaluación, interconsultas, mantenimiento periodontal, control de biopelícula, drenaje de abscesos, raspaje y alisado radicular a campo cerrado y atención de urgencias, de tal forma que las opciones propuestas en la sábana de situaciones anómalas no se detectaron y no se pudieron evaluar para ser tipificadas (figura 1). La única condición reportada en este aspecto consistió en la avulsión de un diente durante la realización de sondaje periodontal, la cual fue clasificada como complicación, como ya se explicó. Además, en algunos casos solo se realizó diligenciamiento de historia clínica y no hubo continuidad en las citas por parte del paciente asociado a dificultad en asistencia por motivos laborales o disponibilidad económica.

En la frecuencia de EA clínicos prevenibles (16,9\%) se encontró una baja incidencia, de los cuales el más común fue la exposición de injerto óseo en bloque; las demás situaciones se registraron con un único caso, aunque se encuentran principalmente relacionados con procedimientos regenerativos (figura 6). Debido a que las situaciones expuestas pudieran ser consideradas complicaciones o errores, se realizará una explicación de los casos para justificar su tipificación, teniendo en cuenta el concepto de EA que se ha descrito y, por otro lado, la consideración de que el suceso de un EA ocurre a consecuencia del paso y no prevención de procesos defectuosos que favorecen su aparición. Lo anterior a partir del modelo explicativo del queso suizo creado por James Reason, en 1990, el cual formula que para que se produzca un daño, es necesario que se alineen los diferentes errores en los diferentes procesos, a semejanza de los orificios del queso, cuando alguno de ellos no lo hace, el daño no se produce [16].

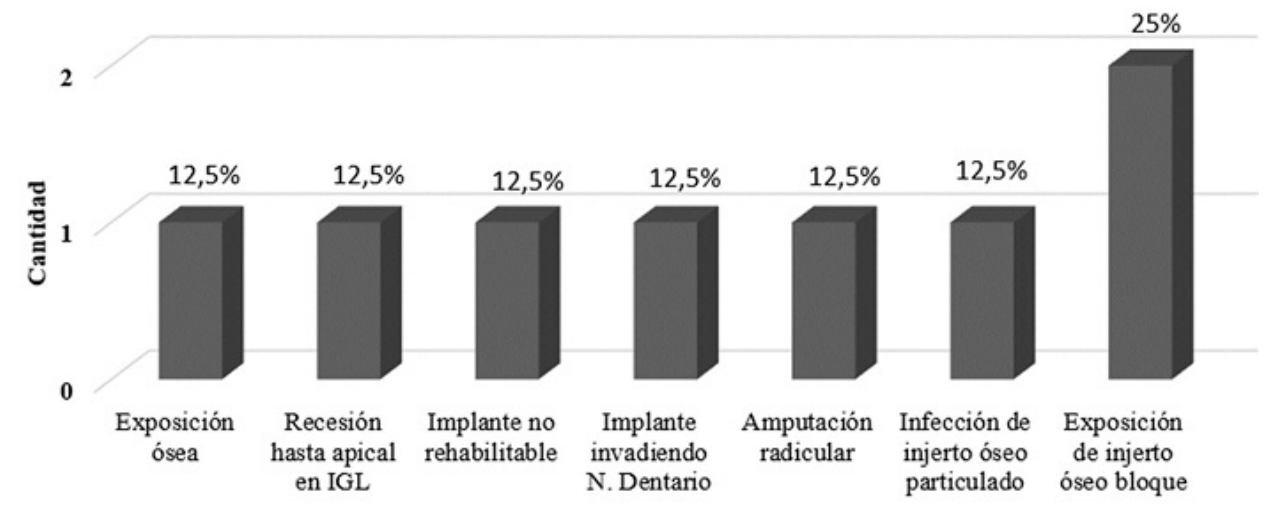

FIGURA 6

Frecuencia de situaciones caracterizadas como evento adverso clínico prevenible IGL: injerto gingival libre, N: nervi

El primer evento sobre la exposición ósea ocurrió a consecuencia a una gingivectomía en un cuadrante que presentaba un implante dental y se expuso hueso del implante. Esto se trató con Fitostimoline ${ }^{\oplus}$ y el paciente asistió 15 días después refiriendo un dolor intenso y recesiones gingivales. Se le diagnosticó secuestro óseo y se le realizó una cirugía para escisión del secuestro, lo que generó múltiples procesos quirúrgicos para disminuir el impacto de las secuelas estéticas y funcionales, principalmente sobre el implante dental y su restauración; adicionalmente, no se encontró seguimiento radiográfico y en la evolución quirúrgica no se describieron cuáles parámetros anatómicos con el hondeo se tuvieron en cuenta o la justificación de la extensión de la gingivectomía. 
El segundo evento correspondió a una recesión que se produjo por un injerto gingival libre, a fin de cubrir una recesión de 31 y 41, cuya evolución no describió la posición de la cresta ósea, que es uno de los criterios para prever el éxito del cubrimiento radicular; sin embargo, a los 3 meses el paciente presentó fístula en la zona injertada, que se remitió al servicio de endodoncia, donde respondieron que la causa no era endodóntica, no se continuó el seguimiento y a los 11 meses presentó recesión del margen gingival hasta el ápice. En este punto se remitió a rehabilitación oral para la realización de una placa estabilizadora oclusal y endodoncia sin justificación del complemento de esta especialidad. La historia clínica finaliza sin reporte de más evoluciones, y se desconoce la resolución clínica y funcional de los dientes 31 y 41.

El tercer evento se generó después de una regeneración ósea en el bloque en maxilar superior, en la zona posterior derecha, en la que se determinó un tiempo de espera de 8 meses para realizar la colocación de implantes dentales; sin embargo, la paciente refirió su intención de que estos fueran colocados a los 5 meses. A pesar de la recomendación del docente y del residente, la paciente insistió. Se solicitó, entonces, una prueba de guía radiográfica/quirúrgica, la cual no se ejecutó, y a las 2 semanas ( 5 meses y medio) se llevó a cabo la cirugía con la colocación de uno y no 4 implantes, como se disponía, sin la justificación descrita. Las evoluciones continuaron su registro un año después, en el que se reportó mal posicionamiento del implante y se solicitó su retiro (explante), por no ser rehabilitable.

En otro caso, se colocó un implante y en el control posquirúrgico la paciente refirió hormigueo y sensación de quemazón. Se le tomó una radiografía y se observó posicionamiento en el canal dentario, por lo cual se decidió el explante. Al control a 3 meses, la paciente refirió ausencia de hormigueo y recuperación de sensibilidad en la zona. Aunque el daño nervioso fue transitorio, no se menciona el uso de la guía quirúrgica o medidas de trabajo de las fresas de preparación del lecho, de acuerdo con los límites anatómicos vistos en la radiografía. Ello evidencia fallas en el proceso de preparación del caso que llevaron a que en la ejecución del procedimiento se presentara la lesión.

Para la situación en la que se realizó raspaje y alisado radicular a campo abierto de los dientes 17 y 16, se encontró un defecto óseo avanzado por palatino del 16 y furca grado II de 16 y17 distal y mesial, respectivamente. Por ello, en un segundo tiempo quirúrgico se realizó la amputación radicular palatina del diente 16, se cureteó el alvéolo de la raíz palatina, se colocó un injerto óseo particulado y se hizo un ajuste oclusal. Posteriormente, se encontraron los dientes 16 y 17 con movilidad grado II, que evidenció una afectación más extensa en la pérdida ósea, por lo cual se determinó mal pronóstico.

En contraste con lo mostrado, cuando sucede una infección de un injerto óseo particulado, ello se clasifica como una complicación, si sucediera por efecto de la enfermedad subyacente del paciente; sin embargo, en este paciente se llevó a cabo una regeneración ósea guiada y después del primer control, sin signos de infección, no hubo un programa de autocuidado con las indicaciones de no usar la prótesis parcial removible. Ello le ocasionó un trauma en el área quirúrgica, que llevó a ruptura de las suturas, exposición del injerto y contaminación. Esto indica fallas en el proceso de atención posquirúrgica, que requiere la misma dedicación, debido a que la ausencia de un seguimiento adecuado puede desencadenar secuelas biológicas irreparables o de mayor complejidad que precisen múltiples intervenciones.

Finalmente, en dos casos se presentó exposición del injerto óseo en bloque, que podría también considerarse una complicación; no obstante, a estos pacientes no se les retiró el injerto inmediatamente, sino que se realizaron otras medidas paliativas, como producir sangrado en la zona, aplicar Fitostimoline ${ }^{\circ} \mathrm{u}$ otro tipo de geles cicatrizantes e injertar tejido conectivo, con el fin cerrar la herida por segunda intención y preservar el injerto óseo. Esto resultó en un manejo inadecuado de la complicación, ya que conllevó más daños y zonas de morbilidad en el paciente como abscesos, inflamación moderada a severa, contaminación de materiales regenerativos, pérdida completa del injerto óseo, necesidad de reinjertar y pérdida de tejido queratinizado. Estas constituyen situaciones que pudieron ser evitadas removiendo el injerto como se aconseja por los protocolos clínicos basados en la literatura. 
Una vez identificadas las fallas en los procedimientos clínicos, se determinó que también es vital identificar falencias administrativas, debido a que podría mediar un daño clínico en el paciente y constituirse como EA no clínicos. Lo anterior se asocia con que la falta de precisión en la descripción completa de las evoluciones, alteraciones en la cronología, carencia de fimas de docente/aprobación del docente inducen a una comprensión inadecuada del proceso clínico-quirúrgico del paciente, siendo un riesgo potencial en una institución universitaria en la que los residentes rotan o finalizan anualmente su ciclo académico y la única evidencia de seguimiento para quien recibe el caso son los datos consignados en la historia clínica.

\section{DISCUSIÓN}

Con la intención de responder a las necesidades expuestas por la Organización Mundial de la Salud sobre la atención segura del paciente, se inició un proceso de reconocimiento de EA en el Posgrado de Endodoncia de la FOPUJ, cuyos resultados demandaron la integración de la iniciativa de otras especialidades, como periodoncia, con el fin de realizar una identificación de fallas clínicas y administrativas que permitan gestionar medidas que no solo incentiven el reporte y seguimiento de EA, sino también minimicen su aparición $[4,7,8]$. Continuando con esta línea de investigación, nuestro estudio se realizó para caracterizar y cuantificar los EA presentados en la Clínica del Posgrado de Periodoncia de la FOPUJ durante el periodo 2011-2012.

En consecuencia, la alerta sobre esta temática adquiere relevancia, debido a que las entidades de salud reportan un incremento en la incidencia de EA derivados del área de odontología, que osciló entre el 3,2 \%y el $16,6 \%$ en la última década; mientras que aquí se encontró en un $26,3 \%$ de las historias revisadas, lo que constituye un aumento significativo con respecto a los promedios mundiales $[17,18]$.

No obstante, los resultados de esta investigación no pueden compararse de forma integral con hallazgos en la literatura, debido a que se encuentran pocos estudios acerca de todos los EA posibles que puedan ocurrir en tratamientos periodontales de rutina y quirúrgicos. En efecto, se reportan de acuerdo con la intervención que se prueba, lo cual genera una ambigüedad errada en la terminología entre complicación, incidente y EA $(9,10)$. Por ende, esta publicación se convierte en la primera nacional y mundial en realizar una caracterización de EA en periodoncia.

Adicionalmente, Ioannidis y colaboradores [19] evaluaron EA en estudios clínicos aleatorizados de intervención terapéutica y concluyeron que no se registró ninguno y en otros no fueron evaluados. Ello genera un vacío en el reporte de estos en la literatura, con resultados coincidentes de Faggion y Giannakopoulos [9] al evaluar ensayos clínicos controlados en periodoncia e implantología oral.

Similarmente, dentro de los hallazgos iniciales del estudio no se encontraron reportes de situaciones identificadas como EA, por lo cual fue necesaria una aclaración conceptual de la terminología de la atención segura del paciente y se creó un formato con posibles situaciones anómalas, entendidas como circunstancias en las que no se obtuvo el resultado adecuado en el tratamiento y que pudieran conllevar un daño del paciente, para ser tipificadas por expertos. Por consiguiente, una reunión con un comité de expertos del Posgrado de Periodoncia de la Facultad de Odontología contrastó la conceptualización de atención segura del paciente con las situaciones anómalas detectadas en las historias y las predefinidas según la literatura.

Así, con la intención de aclarar la tipificación de EA, opuesto a la literatura en la interpretación de respuestas medicamentosas como intolerancia digestiva o reacciones alérgicas cutáneas, según Winkel y colaboradores [20], quienes reportaron 11 EA $(22,4 \%)$ relacionados con el consumo de amoxicilina combinado con metronidazol para periodontitis severa generalizada con terapia básica periodontal en 49 pacientes, y aunque no se registraron respuestas alérgicas o digestivas a medicamentos en este estudio, el comité de expertos determinó que serían consideradas una iatrogenia del tratamiento, al estar comprobado el efecto positivo del apoyo de la antibioticoterapia en el raspaje y alisado radicular a campo abierto.

Adicionalmente, al evaluar el desarrollo de EA asociados a anestésicos locales, los resultados de este estudio no mostraron ninguna situación que correlacionara las dos variables analizadas, por lo que los hallazgos de Pandit y colaboradores [21], mediante el reporte de un ligero enrojecimiento posterior al uso de anestésico, no son comparables, teniendo en cuenta que los autores reportan como EA una 
complicación. Esto evidencia el uso indiscriminado a la referencia de EA como complicación, cuando son dos términos diferentes.

Por otro lado, de las historias evaluadas, 210 correspondieron a tratamientos de interconsulta sin presencia de situaciones anómalas, lo cual demostró un adecuado sistema de referencia de pregrado a posgrado, o entre especialidades, para resolver la condición del paciente. Esto fue contrario a los hallazgos de Darby y colaboradores [22], quienes concluyeron en relación con una dificultad de los odontólogos generales acerca de los factores que influencian el diagnóstico y el tratamiento de la enfermedad periodontal, lo que limita la adecuada respuesta del paciente a un procedimiento.

Con respecto a los EA clínico-quirúrgicos, se presentaron limitaciones para la comparación con este estudio, por cuanto la terminología utilizada por la literatura, al referenciar situaciones como EA cuando representan procesos biológicos de cicatrización como son edema, dolor, enrojecimiento, como se evidencia, según Barreras y colaboradores [23], quienes aplicaron Tisuacryl para el evitar el uso de suturas después de procedimientos quirúrgicos basándose en el desarrollo de EA asociados a su uso, como dolor, hiperemia, sangrado y dehiscencias correspondiendo realmente a complicaciones y no EA; sin embargo, en los resultados de nuestro trabajo solo se reportó un caso de encapsulamiento de suturas y dos con ruptura o pérdida de sutura, las cuales fueron catalogadas dentro de la reunión de expertos como complicación.

Así mismo, el trabajo de Tatakis y Trombelli [24] acerca de los EA como dolor, exudado, edema y desprendimiento del tejido, posterior a tratamientos de regeneración tisular guiada en recesiones gingivales con ácido poliláctico, no fue comparable con las historias revisadas, debido a que no se reportó el uso de ácido poliláctico en los tratamientos periodontales, y solo hubo una fístula posterior a injerto gingival libre, denominado complicación [24].

En cuanto a complicaciones con implantes dentales, Mau y colaboradores [25] presentaron un reporte de caso en el cual describieron un EA asociado con un procedimiento de regeneración ósea guiada postexodoncia con colocación de implante mediato, en el cual a los 4 meses se observó un tono metálico a través de la encía vestibular de los implantes, en el que se encontró una dehiscencia vestibular como consecuencia de una reabsorción ósea severa; sin embargo, es muy difícil establecer si lo reportado en el artículo corresponde a un EA como tal, dado que los autores no explican la condición del alvéolo, la posición tridimensional del implante inmediatamente después de la cirugía y el espesor del gap entre la pared ósea vestibular y la superficie del implante, los cuales son factores que intervienen en la cicatrización ósea alrededor de un implante. En contraste, en las historias evaluadas no se reportan estos hallazgos en las fases quirúrgicas y para las segundas fases se programan con el uso de radiografías periapicales; por ende, solo este tipo de complicaciones reportadas por los autores se detectan tardíamente.

Para los EA de explante durante toma de impresión e implante no rehabilitable identificados aquí no se encontró evidencia científica relacionada con estas situaciones, no siendo posible realizar un comparativo.

También los resultados del presente trabajo demuestran que la población estudiantil desconoce la definición de los términos de atención segura, al registrar en las evoluciones cada situación como un hallazgo sin denominación en la taxonomía, opuesto a lo reportado por Hiivala y colaboradores [26], quienes publicaron los resultados de encuestas realizadas a odontólogos finlandeses en 2010 sobre la seguridad del paciente en los diferentes procedimientos odontológicos. Los autores encontraron que solo un tercio de los odontólogos reportó uno o más incidentes en su consulta en los últimos 12 meses, y al menos una quinta parte de los encuestados había presentado un EA; solo un $16 \%$ admitió un casi EA [26]. Así, una vez tipificado en la FOPUJ, de 929 historias evaluadas entre 2011 y 2012, solo un 5,7 \% de los casos reportados representaron principalmente complicaciones ( $\mathrm{n}: 18 ; 33,9 \%$ ) y EA (n: 15; 26,3 \%). No se identificaron casi $\mathrm{EA}$, por lo que se puede inferir que el reporte de EA clínico-quirúrgicos y no quirúrgicos es bajo, posiblemente asociado a una presión subyacente a medidas legales o señalamiento entre profesionales. 
De igual forma, es importante denotar y hacer una alerta al área administrativa y clínica con respecto a las evoluciones completas y de seguimiento del paciente, sobre todo en procedimientos quirúrgicos, debido a que se encontró que de 1738 tratamientos solo el 23,3 \% registraba controles, lo que genera un subregistro de las posibles secuelas o éxito y, por ende, de la atención segura del paciente. Por otro lado, se encontró en las evoluciones la descripción de cicatrización adecuada posterior al reporte de una alteración durante el proceso de seguimiento, lo que corresponde a una representación inexacta y dificultad, debido a que no se identifica si la resolución fue del tejido sin secuelas o al tratamiento realizado posterior al daño, o si se produjo o no un daño permanente.

Por consiguiente, son precisos planes de seguridad en atención al paciente que puedan establecer un puente de comunicación entre el personal clínico y administrativo que fortalezca la cultura de reporte en pro de garantizar una mejor calidad de servicio al paciente.

\section{CONCLUSIONES}

Dado que de los contextos identificados en las evoluciones ninguno fue considerado bajo alguna denominación, sino un hallazgo, en este estudio se determinó que para identificar un EA en periodoncia hay que basarse en la caracterización de resultados clínicos y no clínicos, que llevaron a un desenlace no deseado denominado situación anómala, para tipi有 carlas según las circunstancias relacionadas con el caso para ser evaluadas con respecto al criterio del comité de expertos y a la conceptualización realizada a los términos de atención segura del paciente del Ministerio de Protección Social y Sistema Obligatorio de Garantía de Calidad en Salud en Colombia, lo que permitió establecer una baja incidencia de estos durante los años 2011 y 2012 en la FOPUJ.

Sin embargo, se encontró un porcentaje disminuido del 23,3\% de controles posquirúrgicos, a pesar de haberse realizado 1738 procedimientos que involucraron cirugías desde acceso y visibilidad, hasta regenerativas y plásticas (mucogingivales). Ello indica un subregistro de posibles situaciones que podrían representar un EA. Por consiguiente, se requiere la atención de la institución para detectar las fallas humanas o administrativas que pudieran generarse para crear barreras de control que minimicen los daños en la atención segura del paciente.

Del mismo modo, se observó una alta variabilidad en la caracterización de los eventos, así como las complicaciones, errores e incidentes; por lo tanto, es importante tener en cuenta que un desenlace clínico no deseado en periodoncia no puede ser generalizado dentro de la taxonomía de política del paciente en atención en salud, debido a que puede variar su tipificación dependiendo del diagnóstico, del pronóstico, del tratamiento y del manejo durante el seguimiento, debido a que la práctica de medidas clínicas o administrativas sin solidez en la literatura pueden desencadenar en cualquiera de las fases anteriores potenciales fallas que lleven a un EA.

\section{RECOMENDACIONES}

Establecer una base de datos a partir del Sistema de Información de la facultad con datos de contacto de los pacientes para realizar un estricto seguimiento y vigilancia por parte de la institución y docentes a cargo, para posiblemente crear o gestionar uno o varios horarios que incluyan mañanas y tardes para la atención de mantenimiento de los pacientes.

Fundamentar en el paciente el protocolo de autocuidado previo y posquirúrgico, el cual involucra el uso o la adaptación de prótesis definitivas o transicionales y la necesidad de asistir a las citas de control y seguimiento para conocer los resultados del tratamiento realizado, y así determinar las consideraciones para el cuidado del procedimiento. 
Promover en el cuerpo docente y estudiantil la comprensión de la terminología de atención segura en el paciente, para que una vez suceda una situación no esperada, pueda ser identificada y clasificada dentro de esta, y mejorar el reporte, con el fin de hacer un llamado a todos los clínicos en cuanto a que la historia clínica no se refiere exclusivamente al diligenciamiento de un diagnóstico, pronóstico y plan de tratamiento y que la atención no finaliza con este último, sino con el seguimiento del efecto positivo o negativo de la atención sobre el paciente.

Fomentar la importancia de cultura del reporte, que incluya una descripción completa y detallada de la evolución de cada procedimiento. Así mismo, cuando se presenten EA se realice seguimiento que permita mediar mecanismos de mejoramiento y prevención en el sistema de calidad de salud ofrecido por la FOPUJ.

Crear una lista de chequeo para revisar la evolución de los diferentes aspectos que se van a evaluar en un control posquirúrgico y socializarla con los residentes de primer y segundo año.

Crear un anexo de reporte de EA, para que una vez sea identificado, sea ingresado al sistema de historia clínica odontológica digital, plataforma propia de la universidad, y se genere una alerta para el residente y administrativo que evalúe la historia para recordar el seguimiento detallado en la historia.

\section{Agradecimientos}

Al personal del archivo de historias clínicas y al Comité de Investigación de la Facultad de Odontología de la Pontificia Universidad Javeriana.

\section{REFERENCIAS}

1. Franco A. Como mejorar la seguridad del acto quirúrgico: el papel del cirujano. En: Franco A, editor. Fundamentos de seguridad al paciente para disminuir errores médicos. Cali, Colombia: Universidad del Valle Programa Editorial; 2006. pp. 89-100.

2. República de Colombia, Ministerio de la Protección Social. Revisión bibliográfica nacional e internacional de la terminología en seguridad del paciente. En: Salcedo AM, Santofimio D, Barrera MV, Vargas AC, editores. Herramientas para promover la estrategia de la seguridad del paciente en el Sistema Obligatorio de Garantía de Calidad de la atención en salud. Bogotá, Colombia: Fundación FITEC; 2007. pp. 21-54.

3. Franco A. La seguridad clínica de los pacientes: entendiendo el problema. Colomb Med. 2005 Abr-Jun; 36(2): 130-3.

4. World Health Organization. International classification for patient safety. Geneva, Switzerland; 2007.

5. República de Colombia, Ministerio de la Protección Social, Programa de Apoyo a la Reforma de Salud, Asociación Centro de Gestión Hospitalaria. Sistema Obligatorio de Garantía de Calidad en las instituciones prestadoras de servicios de salud. En: Guerrero J, Restrepo F, Vergel J, Guillot N, editores. Pautas de auditoría para el mejoramiento de la calidad. Bogotá: Imprenta Nacional de Colombia; 2007. pp. 40-2.

6. Kerguelén C. Reporte y análisis de eventos adversos: la necesidad de estructurar sus relaciones. Vía Salud. 2010 Jul; 52(21): 8-15.

7. Hernández S, Laiseca N, Rincón A. Identificación de eventos adversos que se presentaron en los pacientes que asistieron al posgrado de endodoncia de la Pontificia Universidad Javeriana en el primer periodo del año 2007 [trabajo de posgrado en Endodoncia]. Bogotá, Colombia: Pontificia Universidad Javeriana; 2008.

8. Tafur MC, Camacho LD, Mejía SH, González J, Huertas MF. Frecuencia de eventos adversos de la terapia endodóntica y seguimiento de pacientes atendidos en el Posgrado de Endodoncia de L Pontificia Universidad Javeriana (2007-2008). Univ Odontol. 2014 Jul-Dic; 33(71). doi:10.11144/Javeriana.uo33-71.feat

9. Faggion CM, Giannakopoulos NN. Quality of reporting in abstracts of randomized controlled trials published in leading journals of periodontology and implant dentistry: a survey. J Periodontol. 2012 Oct; 83(10): 1251-6. 
10. Sgolastra F, Gatto R, Petrucci A, Monaco A. Effectiveness of systemic amoxicillin/metronidazole as adjunctive therapy to scaling and root planing in the treatment of chronic periodontitis: a systematic review and metaanalysis. J Periodontol. 2012 Oct; 83(10): 1257-69.

11. Leape LL, Lawthers AG, Brennan TA, Johnson WG. Preventing medical injury. Qual Rev Bull. 1993 May; 19(5): 144-9.

12. Reason J. Human error, models and management. West J Med. 2000 Jun; 172(6): 393-6.

13. Aguirre G, Vásquez F. El error médico: eventos adversos. Cir Ciruj. 2006 Jul; 74(7): 49.

14. Ospina J. Seguridad del paciente en atención primaria. Vía Salud. 2011 Oct-Dic; (58): 18-25.

15. República de Colombia, Ministerio de la Protección Social. Lineamientos para la implementación de la política de seguridad del paciente en Colombia. Bogotá: Ministerio de la Protección Social; 2008.

16. Reason J. Human error. New York, NY: Cambridge University Press; 1990.

17. Organización Mundial de la Salud. Memorias de la 55a Asamblea Mundial de la Salud: Calidad de la Atención en Seguridad del Paciente. Ginebra, Suiza: 23 de marzo del 2002.

18. Kohn LT, Corrigan J, Donaldson MS. To err is human: Building a safer health system. Washington, DC: National Academy Press; 1999.

19. Ioannidis JP, Evans SJ, Gøtzsche PC, O’Neill RT, Altman DG, Schulz K, Moher D; CONSORT Group. Better reporting of harms in randomized trials: An extension of the CONSORT statement. Ann Intern Med. 2004 May; 141(10): 781-8.

20. Winkel EG, Van Winkelhoff AJ, Timmerman MF, Van der Velden U, Van der Weijden GA. Amoxicillin plus metronidazole in the treatment of adult periodontitis patients. A double-blind placebo-controlled study. J Clin Periodontol. 2001 Apr; 28(4): 296-305.

21. Pandit N, Gupta R, Chandoke U, Gugnani S. Comparative evaluation of topical and electronic anesthesia during scaling and root planing. J Periodontol. 2010 July; 81(7): 1035-40.

22. Darby IB, Angkasa F, Duong C, Ho D, Legudi S, Pham K, Welsh A. Factors influencing the diagnosis and treatment of periodontal disease by dental practitioners in Victoria. Aust Dent J. 2005 Mar; 50(1): 37-41.

23. Barreras MA, Barreras LM. Aplicación del Tisuacryl como tratamiento alternativo en afecciones estomatológicas. Rev CENIC Cienc Biol. 2006 Mar; 37(3): 147-51.

24. Tatakis DN, Trombelli L. Adverse effects associated with a bioabsorbable guided tissue regeneration device in the treatment of human gingival recession defects. A clinicopathologic case report. J Periodontol. 1999 May; 70(5): 542-7.

25. Mau LP, Cheng CW, Hsieh PY, Jones AA. Biological complication in guided bone regeneration with a polylactic acid membrane: A case report. Implant Dent. 2012 Jun; 21(3): 171-4.

26. Hiivala N, Mussalo-Rauhamaa H, Murtomaa H. Patient safety incidents reported by Finnish dentists; results from an internet-based survey. Acta Odontol Scand. 2013 Nov; 71(6): 1370-7.

\section{Notas}

* Artículo de investigación

Licencia Creative Commons CC BY 4.0

Cómo citar este artículo: Tafur DJ, Ramírez GP, Cárdenas CA, Serrano JJ, Sarralde AL, Camacho SP, Rodríguez A, González J. Características y prevalencia de los eventos adversos presentados en la Clínica del Posgrado de Periodoncia de la Facultad de Odontología de la Pontificia Universidad Javeriana durante el periodo 2011-2012. Univ Odontol. 2016 Jul-Dic; 35(75): 1-13. https://www.doi.org/10.11144/ Javeriana.uo35-75.cpea 